\title{
ON THE TRIAD EXCISION THEOREM OF BLAKERS AND MASSEY
}

\author{
SHÔRÔ ARAKI
}

The purpose of the present paper is to give a new proof to the triad excision theorem of Blakers and Massey [1], in case $m \geqq 2$ and $n \geqq 2$, by the aid of path spaces and in connection with a recent work of J. P. Serre [2].

1. Preliminary. Let $X, A, B$ be topological spaces such that $X \supset A, B$. By $\Omega_{A, B}(X)$ we denote the totality of paths in $X$ which start $A$ and terminate in $B$; an element $(\sigma, I) \in \Omega_{A, B}(X)$ is represented by a continuous map $\sigma: I \rightarrow X$ of the closed unit interval $I$ into $X$ such that $\sigma(0) \in A$ and $\sigma(1) \in B$. Then $\Omega_{A, B}(X)$ is topologized by the compact open topology.

Let $p_{s}$ be the projection of $\Omega_{A, B}(X)$ to $A$ such that for $(\sigma, I) \in \Omega_{A, B}(X)$ $p_{s}(\sigma, I)=\sigma(0)$, and let $p_{t}: \Omega_{A, B}(X) \rightarrow B$ be the projection such that $p_{t}(\sigma, I)$ $=\sigma(1)$ for $(\sigma, I) \in \Omega_{A, B}(X)$.

In the sequel, it is assumed that for a triad $\left(X ; A, B, x_{0}\right)$ and for spaces of paths such as $\Omega_{A, B}(X), \Omega_{A, x_{0}}(X)$, and so on, $X, A, B, A \cap B$, and spaces of paths are all arcwise connected, and that a reference point of any spaces of paths used, is taken to be an element represented by a constant map $e: I \rightarrow x_{0}$.

The following relations are obvious:

(a)

(c)

(d)

$$
\begin{array}{ll}
\pi_{i-1}\left(\Omega_{x_{0}, x_{0}}(X), e\right) \approx \pi_{i}\left(X, x_{0}\right) & \text { for all } i \geqq 1, \\
\pi_{i-1}\left(\Omega_{A, x_{0}}(X), e\right) \approx \pi_{i}\left(X, A, x_{0}\right) & \text { for all } i \geqq 1,
\end{array}
$$

$$
A \text { is a deformation-retract of } \Omega_{A, X}(X) \text {, }
$$

where $\left(X ; A, B, x_{0}\right)$ is a triad.

The above isomorphisms $(a),(b)$ and $(d)$ are referred to as canonical isomorphisms.

Let $(X, A)$ be a pair of topological spaces, i.e., $X \supset A$. Suppose that $X$ is $p$-connected for $p \geqslant 1$ and $\left(X, A, x_{0}\right)$ is $q$-connected for $q \geqslant 1$, then $\Omega_{d, x_{0}}(X)$ is $(q-1)$-connected. $\left(\Omega_{A, X}(X), p_{t}, X\right)$ has a fibred structure in the sense of $\mathrm{J} . \mathrm{P}$. Serre, the fibre of which is $\Omega_{A, x_{0}}(X)$. Considering this fibre space, we have the following exact homology sequence with respect to integer coefficients, following J. P. Serre, [2] Chap. III. prop. 5 p. 468;

Received March 17, 1953. 


$$
\begin{gathered}
H_{p+q}\left(\Omega_{A, x_{0}}(X)\right) \stackrel{h^{*}}{\longrightarrow} H_{p+q}\left(\Omega_{A, X}(X)\right) \stackrel{p_{t}^{*}}{\longrightarrow} H_{p+q}(X) \stackrel{\Sigma^{*}}{\longrightarrow} H_{p+q-1}\left(\Omega_{A, x_{0}}(X)\right) \longrightarrow \ldots \\
\ldots \rightarrow H_{1}\left(\Omega_{A, x_{0}}(X)\right) \longrightarrow H_{1}\left(\Omega_{A, X}(X)\right) \longrightarrow H_{1}(X) \longrightarrow 0
\end{gathered}
$$

where $\sum^{*}$ is transgression.

Now, we define homomorphisms

$$
c_{k}^{*}: H_{k}\left(\Omega_{A, x_{0}}(X) ; G\right) \longrightarrow H_{k+1}(X, A ; G) \quad \text { for all } k \gtrsim 1
$$

by constructing chain maps, where $G$ is an arbitrary coefficient group. For this we use singular cubical homology groups as homology groups defined by J. P. Serre, [2] p. 440.

Let $\left(u^{k}, \varphi\right)$ be a singular cube of $\Omega_{A, x_{0}}(X)$, then $\varphi$ defines a map

$$
\bar{\varphi}: I \times u^{k} \longrightarrow X,
$$

which gives a singular cube $\left(I \times u^{k}, \bar{\varphi}\right)$ of $X$. By the correspondence

$$
c_{k}:\left(u^{k}, \varphi\right) \longrightarrow\left(I \times u^{k}, \bar{\varphi}\right)
$$

and by linearity we get a chain homomorphism

$$
c_{k}: C_{k}\left(\Omega_{A, x_{0}}(X)\right) \rightarrow C_{k+1}(X) \text {.- }
$$

From the following calculations

$$
\begin{aligned}
d \circ c\left(u^{k}, \varphi\right)= & d\left(I \times u^{k}, \bar{\varphi}\right) \\
& =\left(\sum_{i=1}^{k}(-1)^{i+1} I \times\left(\lambda_{i}^{0} u^{k}-\lambda_{i}^{1} u^{k}\right)-0 \times u^{k}+1 \times u^{k}, \bar{\varphi}\right) \\
& =-\left(I \times d u^{k}, \bar{\varphi}\right)-\left(0 \times u^{k}, \bar{\varphi}\right)+\left(1 \times u^{k}, \bar{\varphi}\right) \\
& =-c \circ d\left(u^{k}, \varphi\right)-\left(0 \times u^{k}, \bar{\varphi}\right)
\end{aligned}
$$

where $\left(1 \times u^{k}, \bar{\varphi}\right)$ is a degenerate cube and $\bar{\varphi}\left(0 \times u^{k}\right) \subset A$, and from the fact that if $\left(u^{k}, \varphi\right)$ is degenerate cube, $\left(I \times u^{k}, \bar{\varphi}\right)$ is also degenerated, it is concluded that $c_{k}$ induces the following homomorphism

$$
c_{k}^{*}: H_{k}\left(\Omega_{A, x_{i}}(X) ; G\right) \longrightarrow H_{k+1}(X, A ; G) .
$$

Lemma 1. Let $\left(X, x_{0}\right)$ be p-connected for $p \geqslant 1$, and let $\left(X, A, x_{0}\right)$ be $q$-connected for $q \geqslant 1$. Then

i) $c_{k}^{*}$ are isomorphisms onto for $k \leqq p+q-1$,

ii) $c_{p+q}^{*}$ is a homomorphism onto.

Proof. We consider the following diagram

$$
\begin{aligned}
& H_{p+q}\left(\Omega_{A, x_{0}}(X)\right) \stackrel{h^{*}}{\longrightarrow} H_{p+q}\left(\Omega_{A, X}(X)\right) \stackrel{p_{t}^{*}}{\rightarrow} H_{p+q}(X) \stackrel{\Sigma^{*}}{\rightarrow} H_{p+q-1}\left(\Omega_{A, x_{0}}(X)\right) \stackrel{h_{*}}{\longrightarrow} \ldots \\
& \downarrow c_{p+q}^{*} \quad\left\|p_{s}^{*} \quad\right\| c^{*} \quad \downarrow c_{p+q-1}^{*} \\
& H_{p+q-1}(X, A) \stackrel{\text { ว* }}{\longrightarrow} H_{p+q}(\dot{A}) \stackrel{i *}{\longrightarrow} H_{p+q}(X) \stackrel{j^{*}}{\longrightarrow} H_{p+q}(X, A) \stackrel{\text { ə* }}{\longrightarrow} \ldots
\end{aligned}
$$




$$
\begin{aligned}
& \cdots, \longrightarrow H_{1}\left(\Omega_{A, x_{0}}(X)\right) \longrightarrow H_{1}\left(\Omega_{A, X}(X)\right) \longrightarrow H_{1}(X) \longrightarrow 0 \text {. } \\
& \downarrow c_{1}^{*} \quad\|\quad\| \\
& \cdots \longrightarrow H_{2}(X, A) \longrightarrow H_{1}(A) \longrightarrow H_{1}(X) \rightarrow 0 \text {. }
\end{aligned}
$$

Let

$$
\left(u^{i+1}, \varphi\right) \in C_{i+1}\left(\Omega_{A, X}(X)\right)
$$

be given, then we have

$$
\begin{aligned}
& i \circ p_{s}\left(u^{i+1}, \varphi\right)=(\left.0 \times u^{i+1}, \bar{\varphi}\right) \in C_{i+1}(A) \subset C_{i+1}(X), \\
& p_{i}\left(u^{i+1}, \varphi\right)=\left(1 \times u^{i+1}, \bar{\varphi}\right) \in C_{i+1}(X), \\
& \begin{aligned}
d\left(I \times u^{i+1}, \bar{\varphi}\right)=-\left(I \times d u^{i+1}, \bar{\varphi}\right) & -\left(0 \times u^{i+1}, \bar{\varphi}\right) \\
& +\left(1 \times u^{i+1}, \bar{\varphi}\right) .
\end{aligned}
\end{aligned}
$$

This proves

$$
i^{*} \circ p_{s}^{*}=\iota^{*} \circ p_{t}^{*}
$$

Next, given

$$
\left(u^{i}, \varphi\right) \in C_{i}\left(\Omega_{A, x_{0}}(X)\right),
$$

then we have

$$
\begin{aligned}
\partial \circ c\left(u^{i}, \varphi\right) & =d\left(I \times u^{i}, \bar{\varphi}\right) \\
& =-c \circ d\left(u^{i}, \varphi\right)-\left(0 \times u^{i}, \bar{\varphi}\right) \\
& =-p_{s} \circ h\left(u^{i}, \varphi\right)-\operatorname{cod}\left(u^{i}, \varphi\right) .
\end{aligned}
$$

Thus the identity

$$
\partial^{*} \circ c^{*}=-p_{s}^{*} \circ h^{*}
$$

is established.

By J. P. Serre, [2] p. 469, we get the following equivalent homology sequences :

$$
\begin{aligned}
& \underset{\|}{H_{i+1}\left(\Omega_{A, x_{0}}(X)\right)} \longrightarrow \underset{H_{i+1}\left(\Omega_{A, X}(X)\right) \longrightarrow H_{i+1}\left(\Omega_{A, X}(X), \Omega_{A, x_{0}}(X)\right)}{\| p_{t}^{\prime *}} \\
& \left.H_{i+1}\left(\Omega_{A, x_{0}}(X)\right) \longrightarrow H_{i+1}\left(\Omega_{A, X}(X)\right) \quad \longrightarrow \quad H_{i+1}(X)\right) \\
& \stackrel{\partial *}{\longrightarrow} H_{i}\left(\Omega_{A, x_{0}}(X)\right) \longrightarrow H_{i}\left(\Omega_{A, X}(X)\right) \\
& \stackrel{\Sigma^{*}}{\longrightarrow} H_{i}\left(\Omega_{A, x_{0}}(X)\right) \longrightarrow H_{i}\left(\Omega_{A, x}(X)\right)
\end{aligned}
$$

for $1 \leqq i \leqq p+q-1$, i.e., we have $\sum^{*}=\partial^{*}{ }^{\circ} p_{t}^{*-1}$.

We now consider the following diagram:

$$
\begin{aligned}
& H_{i+1}\left(\Omega_{A, X}(X), \Omega_{A, x_{0}}(X)\right) \\
& \downarrow p_{t}^{* *} \searrow^{2 *} \\
& H_{i+1}(X) \stackrel{\Sigma^{*}}{\longrightarrow} H_{i}\left(\Omega_{A, x_{0}}(X)\right) \\
& j^{*} \searrow \quad \downarrow c_{i}^{*} \\
& H_{i+1}(X, A)
\end{aligned}
$$


Let

$$
\sum_{j}\left(u_{j}^{i+1}, \varphi_{j}\right) \in Z_{i+1}\left(\Omega_{A, X}(X), \Omega_{A, x_{0}}(X)\right)
$$

be given, then we have

$$
\begin{aligned}
& p_{t}^{\prime}\left(\sum_{j}\left(u_{j}^{i+1}, \varphi_{j}\right)\right)=\sum_{j}\left(1 \times u_{j}^{i+1}, \bar{\varphi}_{j}\right) \in Z_{i+1}(X), \\
& \partial\left(\sum_{j}\left(u_{j}^{i+1}, \varphi_{j}\right)\right)=\sum_{j}\left(d u_{j}^{i+1}, \varphi_{j}\right) \in Z_{i}\left(\Omega_{A, x_{0}}(X)\right), \\
& \operatorname{c} \partial\left(\sum_{j}\left(u_{j}^{i+1}, \varphi_{j}\right)\right)=\sum_{j}\left(I \times d u_{j}^{i+1}, \bar{\varphi}_{j}\right) \in Z_{i+1}(X, A) .
\end{aligned}
$$

Consider the following chain

$$
\sum_{j}\left(I \times u_{j}^{i+1}, \bar{\varphi}_{j}\right) \in C_{i+2}(X),
$$

we have

$$
\begin{aligned}
d\left(\sum_{J}\left(I \times u_{j}^{i+1}, \bar{\varphi}_{j}\right)\right) & =-\sum_{j}\left(I \times d u_{j}^{i+1}, \bar{\varphi}_{j}\right)-\sum_{j}\left(0 \times u_{j}^{i+1}, \bar{\varphi}_{j}\right)+\sum_{j}\left(1 \times u_{j}^{i+1}, \bar{\varphi}_{j}\right) \\
& =-\left(c \circ \partial-p_{t}^{\prime}\right)\left(\sum_{j}\left(u_{j}^{i+1}, \varphi_{j}\right)\right)-\sum_{j}\left(0 \times u_{j}^{i+1}, \bar{\varphi}_{j}\right),
\end{aligned}
$$

where $\sum_{j}\left(0 \times u_{j}^{i+1}, \bar{\varphi}_{j}\right) \in C_{i+1}(A)$. This proves

$$
j^{*} \circ p_{t}^{*}=c^{*} \circ \partial^{*},
$$

so that

$$
c^{*} \circ \Sigma^{*}=j^{*} \circ \iota^{*}
$$

has been established.

$(\alpha),(\beta)$ and $(\delta)$ show that it holds some commutativity or anti-commutativity in each tetragon of the firstly mentioned diagram. As $p_{s}^{*}$ is isomorphism onto by $(c)$ and as $\iota^{*}$ is isomorphism onto induced by identity map, by using "five lemma," we get the first conclusion of this lemma.

$(\alpha),(\beta)$ and $(\gamma)$ show that the following diagram is commutative or anticommutative :

$$
\begin{aligned}
& H_{p+q+1}\left(\Omega_{A, X}(X), \Omega_{A, x_{0}}(X)\right) \stackrel{\partial^{\prime *}}{\longrightarrow} H_{p+q}\left(\Omega_{A, x_{0}}(X)\right) \\
& \downarrow p_{t, p+q+1}^{\prime *} \quad \downarrow c_{p+q}^{*} \\
& H_{p+q+1}(X) \stackrel{j *}{\longrightarrow} \quad H_{p+q+1}(X, A) \\
& \stackrel{h *}{\longrightarrow} H_{p+q}\left(\Omega_{A, X}(X)\right) \stackrel{j^{\prime *}}{\longrightarrow} H_{p+q}\left(\Omega_{A, X}(X), \Omega_{A, x_{i}}(X)\right) \\
& \left\|p_{s, p+q}^{*} \quad\right\| p_{t, p+q}^{*} \\
& \stackrel{\text { a* }}{\longrightarrow} H_{p+q}(A) \stackrel{i *}{\longrightarrow} \quad H_{p+q}(X) .
\end{aligned}
$$

By J. P. Serre, [2] Chap. III prop. 5 cor. 1 p. 469 , we have

( $\varepsilon) p_{t, p+q}^{\prime *}$ is an isomorphism onto, and $p_{t, p+q+1}^{\prime *}$ is a homomorphism onto.

Then, by using a "partial conclusion of five lemma," we get the second con- 
clusion of this lemma.

As a collorary of this lemma, we can easily prove the Hurewicz theorem in the relative case.

Lemma 2. Let $\left(X, A, B, x_{0}\right)$ be a triple, then

$$
\pi_{i}\left(\Omega_{A, x_{0}}(X), \Omega_{B, x_{0}}(X), e\right) \approx \pi_{i}\left(A, B, x_{0}\right) \quad \text { for all } i \geq 1 .
$$

Proof. Let us consider the following diagram

$$
\begin{aligned}
& \ldots \longrightarrow \pi_{i}\left(\Omega_{A, x_{0}}(X)\right) \stackrel{j^{\prime}}{\longrightarrow} \pi_{i}\left(\Omega_{A, x_{0}}(X), \Omega_{B, x_{0}}(X)\right) \stackrel{\partial^{\prime}}{\longrightarrow} \pi_{i-1}\left(\Omega_{B, x_{0}}(X)\right) \\
& \left\|k_{A} \quad \downarrow p_{s} \quad\right\| k_{B} \\
& \ldots \longrightarrow \pi_{i+1}(X, A) \stackrel{\partial}{\longrightarrow} \pi_{i}(A, B) \stackrel{i}{\longrightarrow} \quad \pi_{i}(X, B) \\
& \stackrel{i^{\prime}}{\rightarrow} \pi i-1\left(\Omega_{A, x_{0}}(X)\right) \rightarrow \ldots \\
& \| k_{A} \\
& \stackrel{j}{\longrightarrow} \pi_{i}(X, A) \rightarrow \ldots \\
& \cdots \longrightarrow \pi_{1}\left(\Omega_{A, x_{0}}(X)\right) \longrightarrow \pi_{1}\left(\Omega_{A, x_{0}}(X), \Omega_{B, x_{0}}(X)\right) \longrightarrow \pi_{0}\left(\Omega_{B, x_{0}}(X)\right) \longrightarrow \pi_{0}\left(\Omega_{A, x_{0}}(X)\right), \\
& \ldots \rightarrow \pi_{2}(X, A) \rightarrow \pi_{1}(A, B) \rightarrow \pi_{1}(X, B) \rightarrow \pi_{1}(X, A),
\end{aligned}
$$

where the upper sequence is a homotopy sequence of the pair $\left(\Omega_{A, x_{0}}(X), \Omega_{B, x_{0}}(X)\right)$ and the lower sequence is a homotopy sequence of the triple $\left(X, A, B, x_{0}\right) . k_{\text {. }}$ and $k_{B}$ are canonical isomorphisms and $p_{s}$ denotes also the homorphism induced by the projection $p_{s}$.

Firstly, we prove that $\left(k_{A}, p_{s}, k_{B}\right)$ is a homomorphism of the sequences, i.e., that $\partial \circ k_{A}=p_{s} \circ j^{\prime}, i \circ p_{s}=k_{B} \circ \partial^{\prime}, j \circ k_{B}=k_{A} \circ i^{\prime}$.

The identity $j \circ k_{B}=k_{A} \circ i^{\prime}$ is obvious.

Let $\alpha \in \pi_{i}\left(\Omega_{A, x_{0}}(X)\right)$ be given such that a map $f:\left(E^{i}, \dot{E}^{i}\right) \longrightarrow\left(\Omega_{A, x_{0}}(X), e\right)$ represents $\alpha$, then

$$
k_{A} \circ f=\bar{f}:\left(E^{i} \times I, E^{i} \times 0, E^{i} \times 1 \cup \dot{E}^{i} \times I\right) \longrightarrow\left(X, A, x_{0}\right)
$$

is defined by $f$ canonically. The map

$$
\partial \circ k_{A} \circ f=\bar{f} \mid\left(E^{i} \times 0, \dot{E}^{i} \times 0\right) \longrightarrow\left(A, x_{0}\right) \subset(A, B)
$$

is identical to the map $p_{s} \circ j^{\prime} \circ f$, which proves the identity

$$
\partial \circ k_{A}=p_{s} \circ j^{\prime} \text {. }
$$

Secondly, if $\beta \in \pi_{i}\left(\Omega_{A, x_{0}}(X), \Omega_{B, x_{0}}(X)\right)$ is represented by a map

$$
g:\left(E^{i-1} \times I, E^{i-1} \times 0, E^{i-1} \times 1 \cup \dot{E}^{i-1} \times I\right) \longrightarrow\left(\Omega_{A, x_{0}}(X), \Omega_{B, x_{0}}(X), e\right),
$$

$g$ defines canonically a map

$$
\begin{aligned}
\bar{g}:\left(E^{i-1} \times I \times I^{\prime}, E^{i-1} \times I \times 0^{\prime}, E^{i-1} \times 0 \times 0^{\prime}\right. \\
\left.E^{i-1} \times 1 \times I^{\prime} \cup E^{i-1} \times I \times 1^{\prime} \cup \dot{E}^{i-1} \times I \times I^{\prime}\right) \longrightarrow\left(X, A, B, x_{0}\right) .
\end{aligned}
$$


Then $i \circ p_{s} \circ g$ and $k_{B} \circ \partial^{\prime} \circ g$ are the following restrictions of $\bar{g}$ respectively:

$$
\begin{aligned}
i \circ p_{s^{\circ}} \circ=\bar{g} \mid\left(E^{i-1} \times I \times 0^{\prime}, E^{i-1} \times 0 \times 0^{\prime}, E^{i-1} \times 1 \times 0^{\prime} \cup \dot{E}^{i-1} \times I \times 0^{\prime}\right) & \\
& \longrightarrow\left(A, B, x_{0}\right) \subset\left(X, B, x_{0}\right), \\
k_{B^{\circ}} \circ \hat{o}^{\prime} \circ g=\bar{g} \mid\left(E^{i-1} \times 0 \times I^{\prime}, E^{i-1} \times 0 \times 0^{\prime}, E^{i-1} \times 0 \times 1^{\prime} \cup \dot{E}^{i-1} \times 0 \times I^{\prime}\right) & \longrightarrow\left(X, B, x_{0}\right) .
\end{aligned}
$$

A homotopy between two maps $i^{\circ} p_{s} \circ g$ and $k_{B} \circ \partial^{\prime} \circ g$ will be given in $\left(E^{i-1}\right.$ $\left.\because I \times I^{\prime}\right)$ as follows:

$$
G_{\theta}\left(E^{i-1} \times I \times I^{\prime}\right)= \begin{cases}\bar{g} \mid\left(E^{i-1} \times t \times 2 \theta t\right) & 0 \leqq \theta \leqq 1 / 2, \\ \bar{g} \mid\left(E^{i-1} \times(2-2 \theta) t \times t\right) & 1 / 2 \leqq \theta \leqq 1 .\end{cases}
$$

This proves the identity

$$
i \circ p_{s}=k_{B} \circ \partial^{\prime}
$$

It follows that $\left(k_{A}, p_{s}, k_{B}\right)$ is a homomorphism of the sequences. Since $k_{A}$ and $k_{B}$ are isomorphisms and since $\left(k_{A}, p_{s}, k_{B}\right)$ is a homomorphism of the sequences it is concluded in virtue of "five lemma" that $p_{s}$ also is isomorphism.

(g.e.d.)

Let $\left(X ; A, B, x_{0}\right)$ be a triad, then $\left(\Omega_{X, x_{0}}(X) ; \Omega_{A, x_{0}}(X), \Omega_{B, x_{0}}(X), e\right)$ is also a triad, where $\Omega_{A, x_{0}}(X) \cap \Omega_{B, x_{0}}(X)=\Omega_{A \cap B, x_{0}}(X)$. The following lemma can be proved easily by considering homotopy sequences of each triads and by the above lemma anci by "five lemma."

Lemma 3. Let $\left(X ; A, B, x_{0}\right)$ be triad, then

$\pi_{i}\left(X ; A . B, x_{0}\right) \approx \pi_{i}\left(\Omega_{X, x_{0}}(X) ; \Omega_{A, x_{0}}(X), \Omega_{B, x_{0}}(X), e\right) \quad$ for all $i$ 玉 2 .

LeMMA 4. Let $\left(X ; A, B, x_{0}\right)$ be a triad such that

$X=($ Int $A) \cup($ Int $B)$, and let $(A, A \cap B)$ be $n$-connected $(n \geqslant 1)$, then $(X$, $B)$ is n-connacted.

Proof. Let $\alpha \in \pi_{m}(X, B)$ be represented by a map

$$
f:\left(E^{m}, E^{m-1}, J^{m-1}\right) \longrightarrow\left(X, B, x_{0}\right),
$$

where $m \leqq n$. If we put $U=f^{-1}($ Int $A)$ and $V=f^{-1}(\operatorname{Int} B)$, then $\{U, V\}$ is an open covering of $E^{m}$.

We subdivide $E^{m}$ simplicially such that the mesh of this subdivision is smaller than the Lebesgues number of $\{U, V\}$. Let $K$ and $L_{1}$ be maximal subcomplexes contained in $U$ and $V$ respectively. Let us put $L=L_{1}+E^{m-1}$ $+J^{m-1}$ and $M=K \cap L$, then we have $K \cup L=E^{m}$. Let

$$
g:(K, M) \longrightarrow(A, A \cap B)
$$

be a restriction of $f$. As $K$ is $m$-dimensional, $m \leqq n$, and as $(A, A \cap B)$ is $n$ connected, $g$ is deformable into $A \cap B$ relative to $M$. Denoting this deforma- 
tion by $g_{t}$, we have

$$
\begin{aligned}
& g_{0}=g, \\
& g_{1}(K) \subset A \cap B, \\
& g_{t}|M=g| M \quad \text { for } 0 \leqq t \leqq 1 .
\end{aligned}
$$

We define a deformation $f_{t}$ of $f$ as follows:

$$
\begin{array}{ll}
f_{t} \mid K=g_{t} & \text { for } 0 \leqq t \leqq 1, \\
f_{t}|L=f| L & \text { for } 0 \leqq t \leqq 1 .
\end{array}
$$

This gives a deformation of $f$ into $B$ relative to $L$, which establishes the lemma.

(q.e.d.)

\section{Proof of the triad excision theorem of Blakers and Massey.}

Now we proceed to prove a theorem of A. L. Blakers and W. S. Massey, [1] p. 192, in case $m, n \geq 2$. The theorem is stated as follows.

TheOREM. Let $\left(X ; A, B, x_{0}\right)$ be a triad which satisfies the following con. ditions :

$$
X=(\operatorname{Int} A) \cup(\operatorname{Int} B):
$$

$$
(A, A \cup B) \text { is m-connected, } m \geqq 2 \text {, and }(B, A \cap B)
$$

is $n$-connected, $n \gtrsim 2$;

then the triad $(X ; A, B)$ is $(m+n)$-connected.

A triad with the condition $(a)$ is said to be proper by a denomination of S. Eilenberg and N. E. Steenrod, [3] p. 34. From Lemma $4(X, A)$ is $n$-connected, $n \geqslant 2$, and $(X, B)$ is $m$-connected, $m \geqslant 2$. Therefore $\Omega_{X, x_{0}}(X), \Omega_{A, x_{0}}(X)$, $\Omega_{B, x_{0}}(X)$ and $\Omega_{A_{n} B, x_{0}}(X)$ are all arcwise connected. If $\left(X ; A, B, x_{0}\right)$ is proper, it is obvious that $\left(\Omega_{X, x_{0}}(X) ; \Omega_{A, x_{0}}(X), \Omega_{B, x_{0}}(X) . e\right)$ is also a proper triad. Thus, from Lemma 3 it is sufficient for us to consider the triad $\left(\Omega_{X, x_{0}}(X) ; \Omega_{A, x_{0}}(X)\right.$, $\left.\Omega_{P, x_{0}}(X), e\right)$ instead of the given triad. As $\Omega_{X, x_{0}}(X)$ is contractible, it is sufficient to prove the theorem in a special case where $X$ is contractible.

Proof. As $(X, A)$ is $n$-connected from Lemma 4 , and as $X$ is contractible, $A$ is $(n-1)$-connected. Thus, by Lemma 1 it is seen that

$$
\begin{aligned}
& c_{i}^{*}: H_{i}\left(\Omega_{A \cap B, x_{0}}(A) ; Z\right) \approx H_{i+1}(A, A \cap B ; Z) \\
& \quad \text { for } 0<i \leqq m+n-2, \\
& c_{m+n-1}^{*}: H_{m+n-1}\left(\Omega_{A \cap B, x_{0}}(A) ; Z\right) \longrightarrow H_{m+n}(A, A \cap B ; Z)
\end{aligned}
$$

is a homomorphism onto.

As $(X, B)$ is $m$-connected and $X$ is contractible, we have, from the same Lemma 1 ,

$$
c_{i}^{\prime *}: H_{i}\left(\Omega_{B, x_{0}}(X) ; Z\right) \approx H_{i+1}(X, B ; Z) \quad \text { for all } i>0 \text {. }
$$


From (1), (3) and from the excision theorem in homology theory we have

$$
\begin{aligned}
l_{i}^{*}: H_{i}\left(\Omega_{A \cap B, x_{0}}(A) ; Z\right) \approx H_{i}\left(\Omega_{B, x_{0}}(X) ; Z\right) & \text { for } 0<i \leqq m+n-2 .
\end{aligned}
$$

Next, we consider the following diagram. The commutativity of this diagram is easily seen :

$$
\begin{array}{ccc}
H_{m+n-1}\left(\Omega_{A \cap B, x_{0}}(A) ; Z\right) & \stackrel{l_{m+n-1}^{*}}{\longrightarrow} H_{m+n-1}\left(\Omega_{P, x_{0}}(X) ; Z\right) \\
\downarrow c_{m+n-1}^{*} & & \| c_{m+n-1}^{* *} \\
H_{m+n}(A, A \cap B ; Z) & \stackrel{e_{m+n}^{*}}{\approx} & H_{m+n}(X, B ; Z)
\end{array}
$$

Since $e_{m+n}^{*}$ is an excision isomorphism, and since $c_{m+n-1}^{\prime *}$ is an isomorphism by (3) and since $c_{m+n-1}^{*}$ is a homomorphism onto by (2), we have

$$
l_{m+n-1}^{*}: H_{m+n-1}\left(\Omega_{A \cap B, x_{0}}(A) ; Z\right) \rightarrow H_{m+n-1}\left(\Omega_{B, x_{0}}(X) ; Z\right)
$$

is a homomorphism onto.

$B_{y}(4)$ and (5), and by considering the homology sequence of the pair $\left(\Omega_{B, \gamma_{0}}(X), \Omega_{A \cap B, x_{0}}(A)\right)$ we can prove

$$
H_{i}\left(\Omega_{R, x_{0}}(X), \Omega_{A \cap B, x_{0}}(A) ; Z\right) \approx 0 \quad \text { for } 0<i \leqq m+n-1 .
$$

From (6) and from the Hurewicz theorem in the relative case where $\pi\left(\Omega_{B, x_{0}}(X)\right) \approx 1, \pi_{1}\left(\Omega_{A \cap B, x_{0}}(A)\right) \approx 1,\left(\Omega_{B, x_{0}}(X), \Omega_{A \cap B, x_{0}}(A), e\right)$ is $(m+n-1)$-connected. This is equivalent to the fact that $\left(X ; A, B, x_{0}\right)$ is $(m+n)$-connected.

(q.e.d.)

In an analoguous way as above we can also prove the theorem corresponding to the case where $m \geqslant 2, n=1$, and $(A, A \cap B)$ is $(m+1)$-simple. But it is unnecessarily too long for us to put down here the proof, so that it is omitted.

We can also prove quite analogously as above a generalization of the triad excision theorem, which has been announced by J. C. Moore [4].

\section{REFERENCES}

[1] A. L. Blakers and W. S. Massey: The homotopy groups of a iriad. II. Ann. of Math., vol. 55 (1952), pp. 192-201.

[2] J. P. Serre: Homologis singuliere des espaces fibres. Ann. of Math., vol. 54 (1951), pp. 425-505.

[3] S. Eilenterg and N. E. Steenrod: Foundations of Algebraic Topology, Princeton Univ. Press, 1952.

[4] J. C. Moore: Bull. Amer. Math. Soc., vol. 58 (1952), Abstract; No. 396.

\section{Mathematical Institute,}

\section{Nagoya University}

